\title{
Weakness of the "missing-stimulus effect" in hooded rats: Gross asymmetry in the Sokolovian orienting response
}

\author{
NIGEL FOREMAN \\ University of Leicester, Leicester, England \\ and \\ CATHERINE THINUS-BLANC \\ CNRS Laboratoire de Neurosciences Fonctionnelles, Marseille, France
}

\begin{abstract}
The conventional Sokolovian orienting response (OR) model predicts that reflexive orientation will be evoked whenever unexpected stimulus change occurs, irrespective of whether the stimulus increases or decreases in intensity. Orientation toward expected but nonappearing stimuli has been termed the "missing-stimulus effect." There are few data to support the existence of the phenomenon, however, and in man, it would appear that the omission of an expected stimulus does not reliably evoke an OR. The present study investigated the "missing-stimulus effect" in hooded rats. Although rats traversing a runway were successfully distracted by unexpected flashing lights presented in the visual periphery at the midpoint of the run, they showed remarkably little response to the omission of "expected" light flashes (to which they had been previously habituated.) The same result was obtained in two further conditions: when the peripheral lights were simply turned off, or when lights that flashed constantly throughout the test session were suddenly omitted. It was argued that orientation to stimulus appearance, and stimulus disappearance, may be mediated by "short-loop" and "long-loop" neural mechanisms, respectively. The latter may be more easily overridden by collateral behaviors than the former. This distinction is consistent with recent modular and neural OR models.
\end{abstract}

The orienting response (OR), first described by Pavlov (1927) and then by Sokolov $(1960,1963,1969,1975)$, was originally thought to be a unitary response, similar in form across species and occurring to any unexpected stimulus. When an OR occurs, sensory receptors are redirected toward the source of potentially useful information, ongoing behaviors are interrupted, and the organism is galvanized for appropriate action if necessary. Clearly, monitoring and responding appropriately to dynamic change in the environment is crucial to survival in all species, and slow or absent ORs might have implications for survival in many situations (Lynn, 1966; Wurtz \& Goldberg, 1972.) Moreover, since orienting to redundant information would be uneconomical, it is not surprising that the response habituates rapidly on repeated stimulus presentations.

Sokolov $(1960,1963,1969,1975)$ argued that an OR was elicited when incoming information failed to conform to predictions, that is, when a mismatch was detected between actual and expected events. Expectations, accord-

The authors gratefully acknowledge the assistance of John Ashworth, Rob Hemmings, Pete Benson, Bill Williamson, and Gordon Evans in constructing and servicing the apparatus and writing software, and that of Dave Mann in his care of the animals. The valuable comments of editorial reviewers are gratefully acknowledged. Correspondence should be addressed to N. P. Foreman, Department of Psychology, University of Leicester, Leicester, LE1 7RH, England. ing to Sokolov, are neural templates or sequential representations against which sensory input is compared. One important prediction from this model is that ORs will be elicited equally well by stimulus change in either increasing or decreasing directions: Sokolov (1963) commented that the OR was a "response to all changes in the stimulus-its application, removal, intensification, weakening, or change of nature" (p. 41), and elsewhere that, following habituation, an OR was reevoked "when the signal is intensified, weakened, lengthened, shortened; when it is presented before the usual time; when it has been omitted at the usual time"' (Sokolov, 1969, p. 674; see Siddle \& Spinks, 1979). There is anecdotal evidence for the elicitation of ORs by absent or disappearing stimuli. For example, a clock that has been ticking regularly but suddenly stops can attract our attention (Voronin \& Sokolov, 1960), and Pribram (1971, p. 51) reported an incident that shows that the absence of an expected, complex stimulus can provoke arousal and orientation. In that case, the nightly run of a noisy locomotive through a small town was suddenly cancelled; at its scheduled time, sleeping residents woke and complained of hearing noises.

The latter example can be accounted for only by assuming that a complex, high-level (perhaps cortical) neuronal model "triggers" orientation when incoming information is incongruous (Pribram, 1971). However, this 
view conflicts with the assumption made by others (Groves \& Thompson, 1970) that orientation is a fundamentally low-level process, organized at the level of the spinal cord. Sokolov $(1963,1975)$ was inclined to implicate the hippocampus of the telencephalon in the triggering of orientation on the basis of perceived mismatches, and supportive electrophysiological data is available for such a hippocampal role (Vinogradova, 1970), although this interpretation has been challenged (Foreman \& Stevens, 1987). Thus, it remains unclear where expectancies might be generated within the CNS and at what level comparison takes place between actual and expected events.

Moreover, the unitary nature of the OR has recently been questioned, since it now appears to be a multicomponent process, involving a series of changes in heart rate (HR), skin conductance (SC), electroencephalographic activity (EEG), and respiration (Barry, 1982, 1984; Grings, 1979) that are partly independent of one another. For example, the rate of habituation of each of these components is different, and the components vary independently with stimulus magnitude, characteristics, and significance (Barry, 1984).

In addition, the "symmetrical" view of the OR has also been challenged by Barry (1984). Earlier authors had generally assumed that unexpected decreases in stimulus intensity would elicit ORs as effectively as increasing intensities (Groves \& Thompson, 1970; Voronin \& Sokolov, 1960), a phenomenon that has become known as the "'missing-stimulus effect"' (Barry, 1984, p. 133). However, Barry points out that "strong support for the existence of this phenomenon is lacking in the literature," and, in the few laboratory studies that have addressed the issue, orientation to diminished or absent stimuli is remarkably weak compared with the brisk ORs that occur to increasing stimulus intensities. Barry $(1984$, p. 133) reports finding in an unpublished study of his own that, at best, "small electrodermal ORs may be elicited in some subjects by the omission of a stimulus after its regular presentation." Indeed, after 20 presentations of an auditory stimulus, only 22 out of 48 human subjects ( $46 \%$ ) showed any evidence of an electrodermal response when that stimulus was suddenly omitted. Siddle and Heron (1975) had previously obtained a similar result (36\%) using the same measure. Clearly, ORs to different types of stimulus may be mediated by separate neural processes that operate with different criteria and constraints.

In the present study, we examined the missing stimulus effect in hooded rats. Irrelevant visual stimuli, presented in the peripheral visual field while animals traversed a runway, (a) appeared unexpectedly, (b) failed to appear when expected, or (c) disappeared unexpectedly.

In all cases, appearing stimuli produced clear ORs but disappearing or absent stimuli usually failed to do so.

\section{METHOD}

\section{Subjects}

The subjects consisted of 15 male Lister hooded rats, taken randomly from breeding stock and weighing between 280 and $320 \mathrm{~g}$ at the start of the experiment. They were housed in standard laboratory cages and allowed ad-lib access to water throughout the study; access to food was restricted to $1 \mathrm{~h}$ per day beginning $30 \mathrm{~min}$ after the final animal had finished testing. Lights in the colony room were on between $7.00 \mathrm{a} . \mathrm{m}$. and 7.00 p.m.

\section{Apparatus}

The apparatus was similar in general construction to that described in an earlier study (Foreman, 1983; see Figure 1). Basically, it consisted of a wooden, gray-painted arena measuring $1 \mathrm{~m}$ square with $40-\mathrm{cm}$-high walls. Recessed by $16 \mathrm{~cm}$, at the midpoint of one end of the apparatus at floor level, was located a top-hinged, Perspex goal door, $6 \mathrm{~cm}$ square, which could be back-illuminated via a standard 12-V stimulus bulb. At the opposite end were two goal doors, identical to that just described (but not recessed), positioned with their centers $25 \mathrm{~cm}$ from the center of the end wall. When any goal door was illuminated, the animals could obtain a small amount of evaporated milk by pressing the illuminated door, which triggered a mechanical dipper mechanism that delivered the milk. Pressing a nonilluminated door produced no reward. Midway between the ends of the apparatus was an infrared photobeam, $5 \mathrm{~cm}$ from the apparatus floor. Distractor bulbs (12-V stimulus bulbs) were located $26 \mathrm{~cm}$ above the apparatus floor on each of the side walls, $34 \mathrm{~cm}$ from the two-door end (referred to hereafter as the discrimination end) of the arena. A small constant voltage was maintained across the distractor bulb filaments, creating a constant, very slight glow. Such warm filaments ensure a rapid stimulus onset when the lights flash. The whole apparatus was controlled on-line via a Commodore PET microcomputer. An overhead video camera, equipped with a wide-angle lens and a videcon tube appropriate for low light levels, was located $2.5 \mathrm{~m}$ above the center of the arena. This was

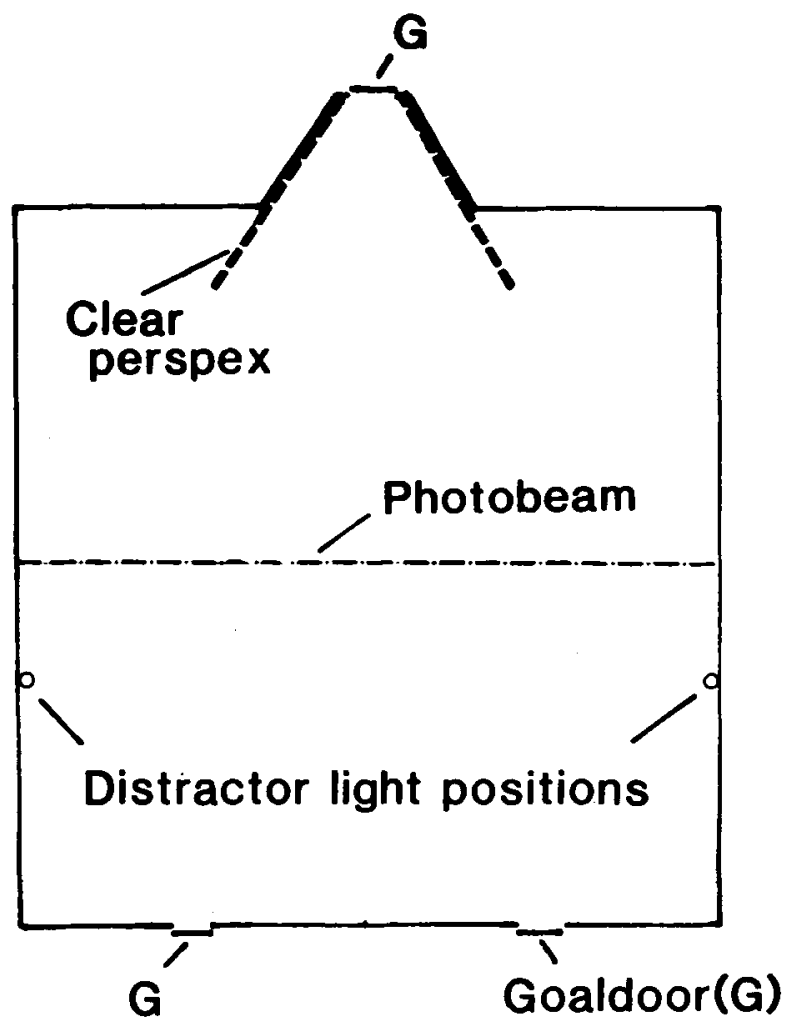

Figure 1. A plan view of the test arena showing the goal door at the nondiscrimination end of the arena and the two goal doors at the discrimination end, and the photobeam and distractor light positions. The clear Perspex guides were added after Phase 1 (between $A$ and $B C$ conditions; see text). 
connected to a video recorder in an adjacent room, from which the animals' behavior in the arena could be observed.

The animals were tested daily between 9:30 a.m. and 4:00 p.m. The test room was illuminated only by a photographic flood light $(150 \mathrm{~W})$ pointed at the white ceiling. This delivered a low, uniform illumination to the surface of the arena. Extraneous sounds were masked by a ventilator fan. Humidity was $60 \%$.

\section{Procedure}

The animals were first pretrained to push goal doors to obtain rewards, all goal doors being constantly illuminated. After the subjects were pressing reliably, their equal experience of the three goal doors was ensured by having reward available only at nonpreferred doors. When all the animals had had experience with pressing all doors, training proper commenced.

Each daily test session began with the animal's being placed in the center of the arena with the single, central goal door illuminated at the nondiscrimination end of the arena. When the animal pressed that door, reward was delivered and the door remained illuminated for $2 \mathrm{sec}$. The light was then extinguished and, without delay, one of the goal doors at the opposite end of the arena became illuminated. The animal had to run to whichever door was illuminated in order to press for reward, delivered as before. Following this, the single goal door at the nondiscrimination end of the arena again became illuminated with zero delay. Thus the animal had to learn to shuttle back and forth between the two ends of the arena. There were $\mathbf{4 0}$ trials ( 80 traverses) per day, making for $\mathbf{4 0}$ light-dark discriminations at the discrimination end in each session. Doorpress errors (presses of the nonilluminated goal door at the discrimination end) were recorded automatically. When this happened, the light was immediately extinguished, and the only way the animal could obtain reward was by running to the opposite end of the arena to initiate the next trial. The animals' run speeds over the second half of each discrimination run were recorded automatically by a timer that started as the rat crossed the photobeam and stopped when either goal door was pressed at the discrimination end of the arena.

The animals' performances were considered stabilized when run speeds were asymptotic and door-push errors were below 10\% (4 or fewer per day) over 2 successive days. The animals were then transferred to the distractor habituation program. On every traverse of the arena toward the discrimination end, both distractor stimulus lights (left and right) flashed twice at a rate of $2 / \mathrm{sec}$ as the animal broke the photobeam at the midpoint of the run. This occurred on each of the 40 discrimination trials per day over 4 successive days; each animal thus experienced a total of 160 double flashes.

At this point, the animals began to be tested for the effects of stimulus omission. Testing took place in five phases. The same animals were tested in each phase, but the groupings were different; group designations were as indicated in the data labeling of Figure 2 (see below).

Phase 1. First, the animals were tested as a single group (A) for 1 day, with the distractor stimuli being omitted on four trials (12, $21,30,37)$. On the first of these four trials, unilateral distractor omission occurred; that is, either left flashes (7 animals) or right flashes ( 8 animals) were omitted. On Trial 30 , the sides on which the distractors were omitted were reversed. On Trials 21 and 37, all distractor flashes were omitted (i.e., bilateral omission). Any disruption caused by distractor omission would have been reflected by increased latencies and by freezing and/or head-turning responses on distractor-omission trials, which were recorded on videotape. The data from this phase are labeled $A$ in Figure 2.

On completion of this part of the study, the animals were renurned to ad-lib feeding for 12 weeks. They were then redeprived and tested in Phase 2, in which modifications of the above procedure were used.
Phase 2. Prior to the commencement of Phase 2, the apparatus had been modified to include two clear Perspex partitions, $32 \times$ $30 \mathrm{~cm}$, that discouraged meandering run paths (Figure 1). Prior levels of runway performance were regained very rapidly by all animals, despite this alteration to the apparatus.

To compare directly the distractibility of appearing and disappearing stimuli, the original group (A) was divided randomly into two groups, B and C, with 8 and 7 rats, respectively. The first group (B) was retrained for 3 days without any distractor flashes, then for 2 days with double-flash distractor lights that appeared on Trials 12 (unilaterally), 20 (bilaterally), 28 (unilaterally), and 37 (bilaterally). The animals that received a left distraction on Trial 12 received a right distraction on Trial 28 , and vice versa. The second group (C) was tested for 2 days as the original group (A) had been, with distractor stimuli present on every trial except for omission trials, which in this case were $12,20,28$, and 37 , that is, the same trials as those used for Group B; the side sequences were the same as those used for Group B. The data for the 2 days of this phase are labeled $\mathrm{BCl}$ and $\mathrm{BC} 2$ in Figure 2.

Phase 3. On the following day, Groups $B$ and $C$ were tested under reversed conditions for a single day. Data for this phase are labeled CB in Figure 2.

Phase 4. Since the previous test conditions involved a dynamic change in one case (appearance, flashes) but no stimulus change in the other (nonappearance, no flashes), a further test session was conducted 2 days later that involved dynamic stimulus change in both conditions. The animals were again reallocated to two groups $(\mathrm{D}, n=7$ and $\mathrm{E}, n=8$ ), drawn equally, as far as possible, from Groups $B$ and C. The animals ran back and forth in the arena as before. For the first group (D), distractor lights remained off throughout testing except that one or both of them would become constantly illuminated on selected trials (Trial 12, unilaterally; Trial 20 , bilaterally; Trial 28 , unilaterally; Trial 37 , bilaterally) as the rat broke the photobeam. These continuous, nonflashing lights were extinguished only when a goal door was pressed. Conversely, for the second group (E), both peripheral bulbs were constantly illuminated prior to the introduction of the animal to the arena, and they remained on until the predetermined omission trials, when one or both were extinguished for the interval between photobeam break and doorpress. The distractor omission sequence-trial numbers, uni- or bilateral side(s) omitted-was the same as the positive distraction sequence used for Group D. The data for this phase are labeled DE in Figure 2.

Phase 5. Two days later, a condition representing a visual equivalent of the "ticking clock" phenomenon (Voronin \& Sokolov, 1960) was run. The animals were once again reallocated to form two new groups $(F, n=7 ; G, n=8)$. To ensure that the animals were still responsive to distractor flashes, the first group (F) was run using a standard distraction paradigm. Thus, a double flash of a distractor light or lights occurred on preset trials (Trial 20, unilateral; Trial 26, bilateral; Trial 33, unilateral; Trial 38, bilateral), but remained off otherwise. For the second group $(G)$, both distractor lights were already flashing at a rate of 2 flashes/sec when an animal was introduced into the arena. Using the same preset trials (and side sequence, etc.) as had been used for Group $F$, one or both of the peripheral distractors would cease to flash for $1 \mathrm{sec}$ (i.e., "miss" two flashes) when the rat broke the photobeam, but then resume flashing until the next preset distraction trial. Data for this phase are labeled FG in Figure 2.

\section{RESULTS}

Throughout, irrelevant peripheral light flashes will be referred to as "distractors"; trials on which these appeared unexpectedly will be referred to as positive dis- 
tractor trials, and trials on which such stimuli were unexpectedly omitted as distractor-omission trials. Thus, in the latter case, a distractor stimulus was distracting by virtue of its nonappearance.

The latency increase occurring on any type of distraction trial was expressed as an index, namely, a multiple of that animal's median no-distraction latency (MNDL; Foreman, 1983) for that particular day. The MNDL is arguably a representative measure of each animal's latency on "routine" trials and an appropriate baseline against which to assess the disrupting effect of distractions; a median, unlike a mean, has the advantage of being relatively unaffected by occasional extreme scores. It was calculated, separately for each animal on each test day, as the median of all trial latencies on that day except for the first trial of the day (the latency for which can show great variability, depending on how rapidly animals settle down to the task), distraction trials, and the trials immediately following distraction trials (which are sometimes also subject to disruption; see below). Thus, in the present study, the MNDL was based on a total of 31 latencies per animal per day. However, when distraction latencies are transformed into multiples of the MNDL (generated by dividing each distraction latency score by that subject's own MNDL for that day), the multiples tend to be distributed with a marked positive skew; therefore, each value is subjected to reciprocal transformation prior to parametric statistical analysis, thus yielding the 1/Mult. MNDL values averaged within groups displayed in Figure 2.

In view of the reallocation method by which groups were composed, and since "bilateral" trials always followed "unilateral" trials, ${ }^{1}$ statistical analyses did not formally compare unilateral and bilateral conditions or include repeated measures across days. Instead, each day was treated as a separate study; first, appearance and disappearance conditions were compared for all distraction trials (averaging across unilateral and bilateral conditions), and then unilateral distraction trials (averaging 1 and 3) and bilateral distraction trials (averaging 2 and 4 ) were analyzed.

In the training stage of the initial study, when distractor stimuli were first introduced to Group A on every trial, all animals showed evidence of orientation; they often froze and/or turned to one of the distractors. They would sometimes approach and investigate a distractor stimulus that appeared on early trials, typically resulting in recorded distraction latencies more than three times their median no-distraction latencies, as has been reported before in control animals (Foreman, 1983; Goodale, Foreman, \& Milner, 1978). Indeed, all animals in this study showed an initial, substantial increase in latency, although they quickly habituated to repeated stimulus presentation. By about Trial 6, their latencies had returned to the asymptotic level recorded on the previous day. Thus, the introduction of a flashing light in the visual periphery was shown to be a powerful stimulus for eliciting a brisk OR, as has been found in many previous studies (see Foreman, 1983; Foreman \& Stevens, 1987; Goodale et al.,
1978; Raphelson, Isaacson, \& Douglas, 1965), but habituation was rapid.

A very different picture emerged when expected distractor stimuli were omitted on selected trials. Condition $\mathrm{A}$ in Figure 2 shows that, in contrast to the results of standard, positive distraction studies (see above), very few ORs $(7 / 60$, or $11.7 \%)$ occurred to omitted distractor flashes. Expressing each distractor-omission-trial latency as a multiple of the MNDL for that day, and taking $>2$ MNDL as the criterion for a distraction's having occurred,

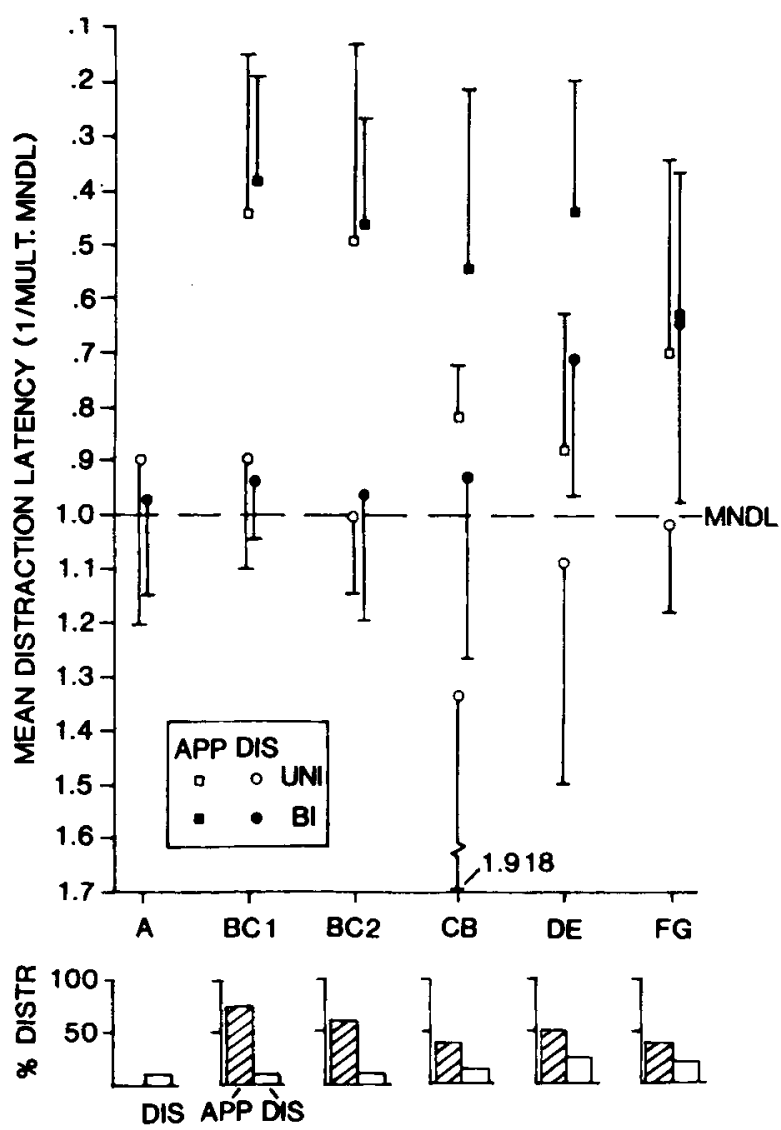

Figure 2. Upper panel: Runway distraction scores in appearance and nonappearance/disappearance distractor conditions. These are transformed latency measures, derived as follows (also see text): each distraction trial latency was expressed as a multiple of that animal's median no-distraction latency (MNDL) for that day and subjected to reciprocal transformation. Scores plotted in the figure are the means and standard deviations of these values for each test condition. Note that the ordinate scale increases in a downward direction. Thus, points vertically above 1.0 represent increases in latency on distraction trials (compared with no-distraction trials). When nodisruption/latency increase occurred on distraction trials, a score of 1.0 would result. Each test condition is labeled according to group designations (see Method), that is, original group (A), Groups B and $\mathrm{C}$ on Days 1 and 2 (BC1 and BC2, respectively), reversal of Groups $B$ and C (CB), and so forth. Filled square symbols represent trials on which distractor stimuli appeared unexpectedly, circles represent unexpected distractor nonappearance or disappearance. Lower panel: Percentages of orientations ( $>2 \times M N D L)$ on appearance (hatched) and disappearance/nonappearance trials in each of the conditions, A to FG. Disappearance/nonappearance distraction trials are labeled DIS. 
only 4 animals showed orienting toward the locus of a "missing" stimulus. Video recordings of the rats' behavior on distraction trials gave identical results.

The same result occurred when Group A was divided into Groups B and C for 2 test days (Figure 2: BC1 and $B C 2$, respectively.) Although ORs to distractor appearance occurred in 24/32 (75\%) of the cases on Day 1 and in $20 / 32(62.5 \%)$ of the cases on Day 2 , in only $3 / 28$ $(10.7 \%)$ of the cases did they occur to omitted distractor lights on each day. The $t$ test (one-tailed) was used to compare the mean reciprocals of Mult. MNDL scores for positive distraction trials in Group B with stimulus omission trials in Group C. Positive distraction produced a significantly greater latency increase on Day $1[t(13)=6.86$, $p<.001$ ], and this result was significant for both unilateral $(t=3.16 ; p<.01)$ and bilateral conditions $(t=6.29 ; p<.001)$. The corresponding analyses for Day 2 were also significant $(t s=4.51,3.27$, and 4.17, respectively; $p s<.005$ ).

When Groups $B$ and $C$ were reversed (Figure 2: CB), despite having had only 11 trials to habituate to a new "distractor absent" environment, the animals now exposed to appearing distractors recorded ORs on 11/28 (39.3\%) of the presentations, and those exposed to omitted distractors did so on only $5 / 32(15.6 \%)$ of the trials. The difference in mean reciprocal latency increase between groups was significant for unilateral and bilateral conditions together $(t=2.63, p<.025)$ and separately $(t \mathrm{~s}=$ 2.07 and $2.12, p s<.05$ ).

$\mathrm{DE}$ in Figure 2 shows the results for Groups $\mathrm{D}$ and $\mathrm{E}$, which were tested with an appearing stimulus (which, after appearing, remained on between photobeam break and door press) or a disappearing stimulus (which, after disappearing, remained off), respectively. When stimulus disappearance occurred bilaterally, there was more evidence of orientation than there had been in previous omission conditions $(8 / 32$ orientations, or $25 \%)$, but again there was considerably more orientation toward an appearing light $(14 / 28$, or $50 \%)$. Mean reciprocal latency increase was greater in the appearing than in the disappearing condition $(t=2.60, p<.025)$, but only in bilateral conditions $(t=1.98, p<.05)$, with the result failing to reach significance for the unilateral condition $(t=1.04$, n.s.).

Finally, in Condition FG, in which standard distractibility (Group F) was compared with the omission of a constantly flashing light (Group G), once again, only 6/28 $(21.4 \%)$ of the disappearing distractors produced orientations as opposed to $12 / 32(37.5 \%)$ on ordinary positive distraction trials. All the former distractions occurred on bilateral omission trials (some occurring in both groups), and latency increase was not significantly different for the bilateral condition $(t=0.12$, n.s.). However, there was a significant group difference $(t=2.19, p<.025)$ on unilateral trials, and the result was significant for unilateral and bilateral trials taken together $(t=1.91, p<.05)$.

Thus, it would appear from Figure 2 that bilateral disappearing stimuli in the DE and FG conditions (both of which involved persisting stimulus change) were relatively powerful as compared with their equivalents in earlier conditions. In view of the consistent effectiveness of bilateral appearing stimuli (filled squares in Figure 2) and the consistent lack of distractibility on unilateral disappearance (unfilled circles), the greater effectiveness of bilateral disappearing stimuli in the later conditions would appear to reflect a genuine interaction between trial type and bilateral-omission potency rather than a confounding order effect.

The overall result of this series of studies is unambiguous; of 208 disappearing distractor stimuli, only 32 (15.4\%) produced criterion ORs, whereas 81/152 (53.3\%) of the appearing distractor stimuli did so.

Further analysis of video recordings of selected trials provided some interesting incidental data. In particular, in Phase 1, for which every trial was recorded, 7 animals showed clear evidence of disruption on some discrimination trials immediately following distractor-omission trials, either pausing at the center of the apparatus just prior to photobeam break or slowing as distractors flashed. Such behavior was uncharacteristic of routine trials for these subjects, and occurred despite the absence of distraction on the previous (omission) trial. The majority of these incidents followed bilateral distractor omissions. Furthermore, on the very few occasions when unilateral omissions gave rise to discernible head turns, these were always made toward the side of the remaining distractor and not toward the locus of the absent one.

\section{DISCUSSION}

These studies represent the first test, to our knowledge, of the missing-stimulus effect in animals (though see a comment by Thompson, Berry, Rinaldi, \& Berger, 1979, p. 37), but it has been sparsely investigated in man, with similar negative results (Barry, 1984; Siddle \& Heron, 1975). The lack of available data is surprising in view of its importance vis-à-vis Sokolov's (1960, 1963, 1969, 1975) influential OR model.

The discovery that stimulus absence does not usually provoke orientation might intuitively seem unspectacular, since a sudden burst of sensory stimulation (and consequent dynamic activity at peripheral receptors) would always seem to be the more potent event, particularly when compared with a nonappearing stimulus (where an unchanging sensory environment is simply maintained). However, at a neural level, this is not so. According to Sokolov's $(1960,1963,1969,1975)$ conventional model, once habituation has occurred to stimulus appearance, the sudden absence or unexpected disappearance of that stimulus ought to produce an equally large internal "burst" of activity in the systems that normally inhibit orientation. Indeed, when a missing stimulus has been reported to be effective, such a burst of central activity can perhaps be misinterpreted as though a sensory event had positively occurred; individuals awoken by the absence of an expected sound report hearing noises rather than "unexpected silence" (cf. Pribram, 1971, p. 51). It is important to note that in each of the conditions tested in the 
present study, the absolute magnitude of decrease (in disappearance or nonappearance conditions) was always equal and opposite to the magnitude of the increase when distractors appeared. Thus, stimulus conditions were symmetrical, if orientation was not.

The result is perhaps reminiscent of the feature positive effect (Hearst, 1978), that is, discriminations in which the presence of a feature signals a positive alternative are more readily learned than those in which a feature signals the negative alternative; there again, the presence (rather than absence) of a distinctive stimulus is most readily attended to and associated with behavioral activation.

Nevertheless, our results might be given other interpretations. It might be argued that lack of orienting in the first condition (Group A) might have occurred because, during pretraining and training, animals had had more experience of the test apparatus without distractors than with them, that is, that stimulus absence was the more "normal" or predictable state for these rats. This is an unlikely explanation, since the animals had become thoroughly habituated to stimulus appearance by the time omission trials were introduced. Moreover, the second study (Groups B and C) rules out this explanation. In that case, for one group (C), all their most recent experience of the test apparatus involved regular distractor appearances, yet they showed few ORs when distractors were omitted. Indeed, the missing-stimulus effect was absent even when $B$ and $C$ groups were reversed, that is, when animals had experienced only 11 trials of habituation to the new stimulus environment prior to the introduction of distraction trials.

The results from Groups D and E show that even when dynamic activity occurs at the retina (in both appearance and disappearance conditions), the missing-stimulus effect fails to appear convincingly; thus, the absence of the effect in groups $\mathrm{A}-\mathrm{C}$ was not due simply to the absence of dynamic retinal activity in stimulus nonappearance conditions.

The final study (Groups $F$ and $G$ ) rules out the argument that rats were "generally nondistractible" in later test sessions. Clearly, some habituation had occurred by that stage, but the asymmetry between the appearance and disappearance conditions was still present. Unlike previous conditions, this test represents a close approximation to the "ticking clock" phenomenon, when thorough habituation occurs over several hundred presentations of a repetitive, punctate stimulus. That ORs did occur on six bilateral omission trials is interesting, yet this still represents a low percentage of orientations and the overall difference between appearance and disappearance conditions was significant. It is perhaps worth noting that the latter condition was the only one in which a stimulus, having disappeared, made a swift reappearance (i.e., following the omission of two flashes). Thus, the ORs that seemed to occur in this condition toward disappearing stimuli may, once again, have been evoked by stimulus appearance-in this case, by flash resumption. If so, this suggests that disappearing stimuli, while not themselves eliciting ORs, can dishabituate orientation toward subsequent stimuli (see Thompson et al., 1979). Further support for this explanation is provided by the present incidental findings that trials on which distractors unexpectedly failed to appear could have a delayed influence on the subsequent trial, and also that on unilateral disappearance trials, attentional movements were made toward the present (rather than the locus of the absent) distractor.

Taken together, the above results cannot be explained by Sokolov's $(1960,1963,1969,1975)$ original formulation for the OR. Expectancies will be equally mismatched by decreases or increases in stimulus intensity, yet stimulus increases are consistently more powerful elicitors of ORs. Nevertheless, the present results are consistent with recent alternative models of orienting behavior. Barry (1984) argues that the components of the OR that reflect stimulus registration and encoding are absent on missing-stimulus trials, and that such trials rely less upon reflexive orienting than upon the activation of orienting via cortical representations of expected eventsperhaps what Maltzman (1979) refers to as "cortical set," guiding "voluntary ORs." Hulstijn (1979) has also commented that "some stimulus changes, mainly comprising changes in gross physical features automatically evoke an OR and automatically attract attention, whereas other types of stimulus change, such as ... the omission of a stimulus, evoke an OR only if the attention of the subject has been focused to the relevant stimulus features"' (p. 563). In common parlance, a stimulus may be said to be conspicuous by its absence, but only in certain circumstances.

Following a review of animal studies of attention, Foreman and Stevens (1987) drew a distinction between shortloop and long-loop orienting processes, arguing that whether orientation will occur depends upon the amount of information needed to identify a stimulus as being worthy of focal scrutiny. Thus, we can begin to speculate about the levels at which information for orienting purposes is processed. In the case of simple repetitive stimuli, short-loop orienting systems may be involved, perhaps at brainstem/spinal levels and involving relatively autonomous processing by structures such as superior colliculus (Foreman \& Stevens, 1987; Goodale et al., 1978). Decerebrate and precerebrate animals show habituation (and sensitization) to such stimuli (Groves \& Thompson, 1970); indeed, several recent studies have shown that orientation and habituation to simple stimuli are relatively unaffected by neocortical excision (see Yeo \& Oakley, 1983). Thus, the view that the OR and its habituation must occur at the neocortical level (Maltzman, 1979; Pribram, 1971) would seem implausible. However, the more sophisticated behaviors involved in context-related orientation in mammals arguably require higher, comparative mechanisms which may involve hippocampus or neocortical structures (Sokolov, 1963, 1969, 1975). Such a distinction between reflexive orientation and more complex investigatory responses was anticipated by $O ' K e e f e$ and 
Nadel (1978; see Foreman \& Stevens, 1987, for a discussion).

Thus, an appearing unexpected stimulus is likely to be processed by short-loop, high-priority orienting systems that are capable of overriding ongoing behaviors, whereas disappearing or nonappearing stimuli are processed via longer, slower loops that do not possess the same authority to interrupt ongoing motivated behaviors (running to goal doors and taking rewards).

Clearly, the terms "orientation" and "attention" have broad meanings, and registration of, and orientation toward, absent but expected stimuli may depend upon a number of situational factors. An object that is not encountered in its familiar place can evoke a great deal of exploratory behavior (Thinus-Blanc, 1986). Moreover, the decision to orient toward a particular stimulus may depend upon a number of complex judgments concerning its significance and informational content (see above and Siddle \& Spinks, 1979). Nevertheless, within a conventional distraction paradigm, it is clear that the predictions following from Sokolov's $(1960,1963,1969,1975)$ OR model are not fulfilled.

\section{REFERENCES}

BARRY, R. J. (1982). Novelty and significance effects in the fractionation of phasic OR measures: A synthesis with traditional OR theory. Psychophysiology, 19, 28-35.

BARrY, R. J. (1984). Preliminary processes in O-R elicitation. Acta Psychologica, 55, 109-142.

Foreman, N. P. (1983). Distractibility following simultaneous bilateral lesions of the superior colliculus or medial frontal cortex in the rat. Behavioural Brain Research, 8, 177-194.

Foreman, N., \& Stevens, R. (1987). Relationships between the superior colliculus and hippocampus: Behavioral and neural considerations. Behavioral \& Brain Sciences, 10, 101-151.

Goodale, M. A., Foreman, N. P., \& Milner, A. D. (1978). Visual orientation in the rat: A dissociation of deficits following cortical and collicular lesions. Experimental Brain Research, 31, 445-457.

GRINGS, W. W. (1979). Interrelationships among components of orienting behavior. In H. D. Kimmel, E. H. Van Olst, \& J. F. Orlebeke (Eds.), The orienting reflex in humans (pp. 305-321). Hillsdale, NJ: Erlbaum.

Groves, P. M., \& Thompson, R. F. (1970). Habituation: A dual-process theory. Psychological Review, 77, 419-450.

Hearst, E. (1978). Stimulus relationships and feature selection in learning and behavior. In S. H. Hulse, H. Fowler, and W. K. Honig (Eds.), Cognitive processes in animal behavior (pp. 51-88). Hillsdale, $\mathrm{NJ}$ : Erlbaum.

Hulstiun, W. (1979). Selective attention and the orienting response. In H. D. Kimmel, E. H. Van Olst, \& J. F. Orlebeke (Eds.), The orienting reflex in humans (pp. 557-564). Hillsdale, NJ: Erlbaum. LYNN, R. (1966). The orienting response. London: Pergamon.

Maltzman, I. (1979). Orienting reflexes and classical conditioning in humans. In H. D. Kimmel, E. H. Van Olst, \& J, Orlebeke (Eds.), The orienting reflex in humans (pp. 323-351). Hillsdale, NJ: Erlbaum.

OKEEFE, J., \& NADEL, L. (1978). The hippocampus as a cognitive map. Oxford: Clarendon Press.
Pavlov, I. P. (1927). Conditional reflexes. London: Oxford University Press.

Prubram, K. H. (1971). Languages of the brain. Englewood Cliffs, NJ: Prentice-Hall.

Raphelson, A. C., Isaacson, R. L., \& Douglas, R. J. (1965). The effect of distracting stimuli on the runway performance of limbic damaged rats. Psychonomic Science, 3, 483-484.

SidDle, D. A. T., \& HERON, P. A. (1975). Stimulus omission and recovery of the electrodermal and digital vasoconstrictive components of the orienting response. Biological Psychology, 3, 277-293.

SidDle, D. A. T., \& SPINkS, J. A. (1979). Orienting response and information-processing: Some theoretical and empirical problems. In H. D. Kimmel, E. H. Van Olst, \& J. F. Orlebeke (Eds.), The orienting reflex in humans (pp. 473-497). Hillsdale, NJ: Erlbaum.

Sokolov, E. N. (1960). Neuronal models and the orienting reflex. In M. A. B. Brazier (Ed.), The central nervous system and behavior (pp. 187-276). New York: Josiah Macy Jr. Foundation.

Sokolov, E. N. (1963). Perception and the conditioned reflex. Oxford: Pergamon.

Sokolov, E. N. (1969). The modelling properties of the nervous system. In M. Cole \& I. Maltzman (Eds.), A handbook of contemporary Soviet psychology (pp. 671-704). New York: Basic Books.

SokoLov, E. N. (1975). The neuronal mechanisms of the orienting reflex. In E. N. Sokolov \& O. S. Vinogradova (Eds.), Neuronal mechanisms of the orienting reflex (pp. 217-235). Hillsdale, NJ: Erlbaum.

Thinus-BlanC, C. (1986). The concept of cognitive maps and its consequences. In $\mathrm{P}$. Ellen \& C. Thinus-Blanc (Eds.), Cognitive processes and spatial orientation in animal and man (Vol. 1, pp. 1-19). Dordrecht: Martinus Nijhof.

Thompson, R. F., Berry, S., Rinald, P. C., \& Berger, T. W. (1979). Habituation and the orienting reflex: The dual-process theory revisited. In H. D. Kimmel, E. H. Van Olst, \& J. F. Orlebeke (Eds.), The orienting reflex in humans (pp. 21-60). Hillsdale, NJ: Erlbaum.

Vinogradova, O. S. (1970). The hippocampus and orientation. In G. Horn \& R. Hinde (Eds.), Short-term changes in the nervous system and behaviour (pp. 95-140). Cambridge: Cambridge University Press.

Voronin, L. G., \& Sokolov, E. N. (1960). Cortical mechanisms of the orienting reflex and its relation to the conditioned reflex. In $\mathrm{H}$. $\mathrm{H}$. Jasper \& G. D. Smirnov (Eds.), The Moscow Colloquium on EEG and Clinical Neurophysiology. Electroencephalography \& Clinical Neurophysiology, Suppl. 13.

WurTz, R. H., \& GolDBERG, M. E. (1972). Activity of superior colliculus in behaving monkey: IV. Effects of lesions on eye movements. Journal of Neurophysiology, 35, 587-596.

YEO, A. G., \& OAKLEY, D. A. (1983). Habituation of distraction to a tone in the absence of neocortex in rats. Behavioural Brain Research, 8, 403-409.

\section{NOTE}

1. Unilateral distractors always preceded bilateral distractors in this study. Because head turning is most evident on unilateral distraction trials (bilateral trials frequently producing freezing in the absence of head-turning), and in view of the pronounced within-session habituation that occurs in such paradigms, unilateral distractor trials were promoted in order to obtain the maximum data on head-turning. In any event, this was not an important factor.

(Manuscript received February 9, 1987; revision accepted for publication July 13, 1987.) 